\title{
The Success/failure System Hypothesis
}

\author{
Dong-Yih Bau
}

\author{
Department of Information Management, Da-Yeh University, \\ 168 University Rd., Dacun, Changhua 51591, Taiwan, R.O.C.
}

\begin{abstract}
Universal laws elucidate a basic understanding of nature. Imagine how marvellous it would be to have universal laws in biology as there are in physics. Herein, we apply Einstein's principle theory as a constraining method to identify a new universal biological law (or principle) called the success/failure system principle. Based on this principle, we develop a principle theory called the success/failure system hypothesis, which suggests that every planet has the potential to evolve into a success/failure system. Then, we describe the implications regarding our place in the universe, the role of universal laws in biology in a final theory, and the current global challenges of sustainability and scientific ethics. This paper concludes with the interdisciplinary interest and scientific significance of this hypothesis.
\end{abstract}

Keywords: Biological law, Final theory, Principle theory, Success/failure system, Sustainability

\section{Introduction}

Mayr $^{13}$ claimed that Darwin's theory of evolution was the beginning of an intellectual revolution that ultimately resulted in the establishment of biology as an autonomous science, separate from physics. Mayr $^{12}$ also noted that universal laws are without exceptions and are spatio-temporally unrestricted. Thus, regardless of scientific significance, biology, as of yet, has no universal laws. The variation, contingency, and complexity of living systems certainly seem to preclude the idea of universal laws in biology ${ }^{7}$. However, Mayr's oblique comment, "There are few if any universal laws in biology"12, leads one to wonder whether there are universal laws in biology.

Some physicists, such as Bohr $^{12}$, Einstein ${ }^{2,5,8}$, Feynman $^{19}$, and Schrödinger ${ }^{17}$, have shown interest in universal laws in biology. For example, Einstein anticipated universal laws in biology and physics when he expressed his scientific interest in the two great mysteries of the universe: (1) "to contemplate the mystery of conscious life perpetuating itself through all eternity" and (2) "to reflect upon the marvelous structure of the universe which we can dimly perceive" ${ }^{2}$. Einstein tried to solve the latter by proposing a physical theory, general relativity, which has since formed the basis for cosmology. Although Mayr $^{12}$ labelled these theoretical physicists as vitalists, their interest in universal laws in biology has driven our research in this area. Our long-term investigation has led us to use Einstein's principle theory ${ }^{2,5}$ to form the success/failure system principle and develop a hypothesis.

\section{Theoretical Background}

Einstein believed that "nature [the universe] is a perfect structure, seen from the standpoint of reason and logical analysis". science is, on the one hand, a comprehension, as complete as possible, of the connection between the sense experiences in their totality, and, on the other hand, the accomplishment of this aim by the use of a minimum of primary concepts and relations ${ }^{, 2,5}$. This suggests that Einstein proposed a system view of his philosophy of science. Take general relativity as an example. Einstein considered all the celestial bodies including the planets, stars, systems of stars, galaxies, galactic clusters, and the whole universe in a consistent and holistic manner. Eventually, he defined general relativity, which provides the choreography for an entwined cosmic dance of space, time, matter, and energy among these celestial bodies ${ }^{8}$.

Thus, we consider what the system view of biology would be. The part-whole relationship and its associated hierarchical structure may be reflected in a system view of biology. In other words, a whole is a system, its part is itself a system, a part of its part is itself a system, and so on. This can also play out in reverse with a whole being a part of a larger system, which is itself a part of a larger system. Thus, the 
part-whole relationship is a key logical concept that permits us to contemplate the universe, aligning with our search for universal laws in biology.

Another tenet of Einstein's philosophy of science is the symmetries of laws 5 . Thus, it is natural to wonder what a symmetry principle of biology would entail. Inspired by his interest in the eternity of life $\mathrm{e}^{2,5}$, we consider life versus death and success versus failure as the key ideas for discussion. Success and failure form a pair in the evolving life-laden universe ${ }^{13,18}$. The term success/failure system describes a system or object that, over time, may survive and succeed, or fail. Such systems include the panorama of life and of human contrivances. A biosphere of a planet is the largest success/failure system of that planet. By considering the dependency relations between the whole and a part in all systems of the life-laden universe, let us define a general fact: if something (the whole) depends on another thing (a part) for its conditions for success, then it depends on that thing for its causes of failure, and vice versa ${ }^{14}$. With discrete mathematical reasoning, the success/failure system principle imposes a partial ordering on the hierarchical structure of the part-whole relationship as well as symmetry between the dependency relations of the conditions for success and the causes of failure (see the "Hypothesis Development" section). Like general relativity ${ }^{8}$, the success/failure system hypothesis provides the choreography for a different but similarly entwined cosmic dance of success, failure, part, and whole in all concrete systems.

\section{Hypothesis Development}

Einstein's principle theory can be summarized as follows: "The intuitive grasp of the essentials of a large complex of facts leads the scientist to the postulation of a hypothetical basic law [principle], or several such laws. From these laws, he derives his conclusions... which can then be compared to experience. Basic laws [principles] and conclusions together form what is called a "theory.",2

To develop the success/failure system hypothesis as a principle theory, the essentials of a large complex set of facts, which have been defined earlier as the general fact, are needed. By considering this general fact, which reflects such mathematical concepts as the dependency relations of the conditions for success and the causes of failure and requires discrete mathematical reasoning, as an axiom, we develop a hypothetico-deductive system to obtain the success/failure system principle. From this principle, we deduce that every planet has the potential to evolve into a success/failure system, like Earth. Thus, we define the success/failure system hypothesis, which applies the discrete mathematics of partial ordering $(\mathrm{PO})^{15}$ to describe the success/failure system and deduce its conclusion. A possible formulation of the success/failure system principle is $\mathrm{PO}$ conditions for success $=\mathrm{PO}$ causes of failure (Fig. $1)$.

Step 1 (Fig. 1): Given the axiom, the general fact that something (the whole) depends on something (a part) for its conditions for success means that it also depends on that thing for its causes of failure, and vice versa. Thus, we develop an axiomatic system regarding the structure of success/failure systems by first considering the relation conditions for success and then the relation causes of failure.

Step 2 (Fig. 1): To support generalizability, we call a system with the property of the general fact a success/failure system. Thus, a simple success/failure system must have an integrative whole that is separate from the operational principles at the lower level, so that it has a two-level structure $(n=2)$, with the upper level depending on the lower level for its conditions for success. Note that the upper level can depend on one-to-many constituent operational principles. Note also that the upper level depends on itself for its conditions for success. These two properties are inherent in these kinds of dependency relations.

Step 3 (Fig. 1): We apply the same reasoning here as in Step 2 to obtain a three-level structure $(n=3)$ from the two-level structure, where each of the constituent operational principles now becomes another integrative whole that can succeed or fail and is expanded downward. To save space, only some forms of the three-level structure are depicted. Once a success/failure system has a three-level structure, there exists an inherent partial ordering representing the dependency relations. In other words, the conditions for success have the properties of reflexivity, antisymmetry, and transitivity ${ }^{15}$. For example, consider a simple partial ordering of A, B, and $\mathrm{C}$ (refer to the three connected dots in Fig. 1). For A, B, and C, each includes its own conditions for success. This is the property of reflexitivity ${ }^{15}$. While A holds B's conditions for success, the reverse is not true; the same applies to the cases of B and C. This directionality shows the property of antisymmetry ${ }^{15}$. Finally, given that A holds B's conditions for success and B holds C's conditions for success, then A includes C's conditions for success, even though A and $\mathrm{C}$ are not adjacent, demonstrating the property of transitivity ${ }^{15}$. A similar reasoning can be successively applied to obtain the four-level structure $(n=4)$, the five-level structure $(n=5)$, and so on to generally cover all possible forms.

Step 4 (Fig. 1): Thus far, we have only considered the dependency relations of the conditions for success. Now, we repeat the previous steps to consider the dependency relations of the causes of 
failure. Thus, the conditions for success and the

\begin{tabular}{|c|c|}
\hline $\begin{array}{l}\text { Step } 1 \\
\text { The general fact }\end{array}$ & $\begin{array}{l}\text { Step } 2 \\
\mathrm{n}=2 \\
\text { Relation }= \\
\text { conditions for success }\end{array}$ \\
\hline $\begin{array}{l}\text { Step } 3 \\
\begin{array}{l}\text { Relation }= \\
\text { Conditions for success } \\
\text { (partial ordering) }\end{array}\end{array}$ & $\begin{array}{l}\begin{array}{l}\text { Relation }= \\
\text { conditions for success } \\
\text { (partial ordering) }\end{array} \\
\begin{array}{l}\text { Relation }= \\
\text { conditions for success } \\
\text { (partial ordering) }\end{array}\end{array}$ \\
\hline $\begin{array}{l}\text { Step } 4 \\
\begin{array}{l}\text { Relation }= \\
\text { conditions for success } \\
\text { (partial ordering) }\end{array}\end{array}$ & $\begin{array}{l}n=2,3,4,5, \ldots \\
\text { Relation }= \\
\text { causes of failure } \\
\text { (partial ordering) }\end{array}$ \\
\hline $\begin{array}{l}\text { Step } 5 \\
\text { PO conditions for success } \\
= \\
\text { PO causes of failure }\end{array}$ & \\
\hline
\end{tabular}

Fig. 1 A graphical depiction of mathematical steps from the general fact to the formulation $\mathbf{P O}$ conditions for success $=\mathbf{P O}$ causes of failure

Legend: • operational principles; dependency relations

causes of failure are two distinct dependency relations with the same partial ordering, which is established on the condition that the general fact is indeed true.

Step 5 (Fig. 1): The above formal-science approach demonstrates that the success/failure system principle captures all possible forms, rather than the contents, of success/failure systems. Therefore, a success/failure system has partial ordering as its form and a symmetrical property for the conditions for success and the causes of failure. This requires a new notation: $\mathrm{PO}$ conditions for success $=\mathrm{PO}$ causes of failure, indicating the same partial ordering for two distinct relations. Thus, the success/failure system principle is a symmetry principle of biology. Accordingly, we articulate a universal principle below.

The success/failure system principle can be described as follows: (1) A hierarchical success/failure system has an $n$-level structure $(n \geq$ 2 ), where each level has its own operations or 
operational principles; (2) Dependency relations between any two consecutive levels exist, such that the lower level includes the conditions for success and the causes of failure of the upper level.

Let us apply the success/failure system principle to Earth. To analyse the biosphere of our planet, important clues in the literature allow us to deduce its structure. This shows that with universal laws, we can confidently deduce a basic understanding without detailed knowledge, even in biology 5 .

The biosphere of our planet is a global ecological system that can succeed or fail to sustain itself. It has a three-level structure consisting of the inanimate matter layer, the (non-human) life layer, and the human layer. The two-level structure consisting of the inanimate matter layer and the life layer is denoted as the ecosystem and the entire structure is denoted as the homo-ecosystem.

Margulis and Sagan ${ }^{11}$ stated that humans play merely a transient and expendable role, and that although we may pollute the environment for our grandchildren and hasten our demise, this does not affect the continuation of the biosphere. Further, Wilson ${ }^{25}$ once stated that if humans were to disappear, the rest of life would flourish, but if the little invertebrates were to disappear, the biosphere may collapse, with almost everything perishing, including humans. These concepts support the following notions that accord with the success/failure system principle of Earth's biosphere: the topmost layer (humans) relies on the lower layers for success or failure, but the bottom layers (inanimate matter layer and life layer) may not depend on the topmost layer.

Now, we consider the success/failure system hypothesis for any celestial body's ordering structure, taking into account the number of levels. Life and biospheres are success/failure systems. A biosphere consisting of one planet evolves continuously. Once it evolves into a life-laden system, a biosphere becomes a success/failure system with a two-level structure $(n=2)$. Its life layer relies on its inanimate matter layer in order to survive (be successful). A biosphere, such as on Earth, may evolve toward a higher-level structure, depending on the chain of events catalysed by the conditions for success or causes of failure. As these events transpire, many forms of life (and even intelligence) come and go accordingly, also following the success/failure system principle, with varying sophistication.

However, the success/failure system principle does not apply to a barren biosphere, where life does not exist yet (like our planet billions of years ago) or never appears, because although it evolves, there is no notion of success/failure. We may say that a barren biosphere has a one-level structure $(n=1)$ where the only level corresponds to the inanimate matter layer, the material basis for planetary evolution. A barren biosphere may evolve into a lifeladen system with a two-level structure $(n=2)$ such that the life layer, the upper level, relies on the inanimate matter layer, the lower level, for its existence. In short, the success/failure system principle (or by extension, its lack) provides a basis for the potential evolution of any planet into a lifeladen system.

This principle demonstrates that every planet has the potential (see $\mathrm{n}=1$ above) to evolve into a success/failure system $(\mathrm{n}=2)$, although, in reality, the chance of doing so may be very low. For example, chance has favoured Earth with its current status, belonging to a system with a three-level structure $(n=3)$ involving life and humans. Note that the success/failure system hypothesis can be applied to all individual celestial bodies, including stars, planets, asteroids, and satellites.

Recently, reports of newly detected exoplanets have been increasing steadily $y^{3,6,22}$. According to Einstein's philosophy of science ${ }^{2,5}$, (astro)biologists may be interested in establishing this primitive hypothesis holistically by contributing to a complete comprehension of elaborate biological content ${ }^{4}$.

\section{Implications}

Our place in the life-laden universe and our existence on Earth are both main concerns of humanity and science $^{21}$. Humanity holds an important place in the life-laden universe as we are at the third level of a three-level structure, in contrast with the vast majority of celestial bodies, which have a one-level structure.

A final theory may elucidate nature's significant intellectual and spiritual impact on humanity: in physics, scientists seek the fundamental principles from which all other scientific principles can in principle be derived ${ }^{23,24}$. However, current research, which seeks to provide a basis for a final theory, tends to make vague statements or prescribe values for the constants of nature in a range more or less favourable to life $\mathrm{e}^{1,20,23,24}$. The above problem with the anthropic principle derives from our lack of knowledge of universal laws in biology. Since life exists, such universal laws in biology, if they exist, may serve as validation tools for a final theory of the life-laden universe. Thus, we propose that physicists may further seek to validate their theories by making and testing mathematical predictions and deductions based on the success/failure system principle.

Current global challenges of sustainability 9.10 .26 and morality ${ }^{16}$ are also hot topics in biology. As the largest system in our planet, the homo-ecosystem is all-inclusive, and if there is one system that we must 
maintain and sustain, it is the homo-ecosystem. Furthermore, since we need to maintain and sustain the biosphere to survive, the biosphere's sustainability is, logically speaking, our ultimate concern.

\section{Conclusions}

A longstanding issue is whether there are universal laws in biology, as there are in physics (i.e. general relativity), and if so, what their scientific significance might be. An implicit fundamental issue of science is the missing link between physics and biology, as indicated by the anthropic principle and biology as an autonomous science. The success/failure system hypothesis (success/failure system), which we developed by applying Einstein's principle theory in an effort to seek universal laws in biology, begins to address these issues. A key contribution of the hypothesis is the elucidation of humanity's place in the life-laden universe and its subsequent need to sustain Earth. Further, the hypothesis facilitates the unification of physics and biology, which is of scientific significance. This may stimulate interdisciplinary interest toward the supreme goal of science as a unity.

\section{Acknowledgments}

The author would like to thank Anthony Abram for editing and proofreading this manuscript.

\section{References}

[1] Azhar, F. Testing typicality in multiverse cosmology. Phys. Rev. D 91, 103534 (2015).

[2] Calaprice, A. The Ultimate Quotable Einstein Princeton Univ. Press: 330, 368, 388, 396, 425 (2010).

[3] Dittmann, J. A. et al. A temperate rocky superEarth transiting a nearby cool star. Nature 544: 333-336 (2017).

[4] Domagal-Goldman, S. D. et al. The astrobiology primer v2.0. Astrobiology 16: 561-653 (2016).

[5] Einstein, A. Ideas and Opinions Bonanza Books: 226, 293 (1954).

[6] Gillon, M. et al. Seven temperate terrestrial planets around the nearby ultracool dwarf star TRAPPIST-1. Nature 542: 456-460 (2017).

[7] Green, S. Revisiting generality in biology: systems biology and the quest for design principles. Biol. Philos. 30: 629-652 (2015).
[8] Isaacson, W. Einstein: His Life and Universe Simon \& Schuster: 220 (2007).

[9] Johnson, C. N. et al. Biodiversity losses and conservation responses in the Anthropocene. Science 356: 270-275 (2017).

[10] Kraaijenbrink, P. D. A., Bierkens, M. F. P., Lutz, A. F. \& Immerzeel, W. W. Impact of a global temperature rise of 1.5 degrees Celsius on Asia's glaciers. Nature 549: 257-260 (2017).

[11] Margulis, L. \& Sagan, D. Microcosmos: Four Billion Years of Evolution from Our Microbial Ancestors Summit Books: 67 (1986).

[12] Mayr, E. This is Biology: The Science of the Living World Harvard Univ. Press: 15, 62 (1997).

[13]Mayr, E. What Makes Biology Unique? Considerations on the Autonomy of a Scientific Discipline Cambridge Univ. Press: 26 (2004).

[14] Polanyi, M. Personal Knowledge: Towards a Post-Critical Philosophy The Univ. of Chicago Press: chs. 11 and 13 (1958).

[15] Rosen, K. H. \& Krithivasan, K. Discrete Mathematics and Its Applications, 7th ed. McGraw-Hill: 556-558, 597 (2013).

[16] Ruse, M. \& Wilson, E. O. Moral philosophy as applied science. Philosophy 61: 173-192 (1986).

[17] Schrödinger, E. What is Life? Cambridge Univ. Press: chs. 6 and 7 (1944).

[18] Simpson, G. G. The nonprevalence of humanoids. Science 143: 769-775 (1964).

[19] Sykes, C. No Ordinary Genius: The Illustrated Richard Feynman W. W. Norton \& Company: 250 (1994).

[20] Totani, T. Gravity with free initial conditions: a solution to the cosmological constant problem testable by CMB B-mode polarization. Phys. Rev. D 96, 084062 (2017).

[21] Turner, M. S. The power of curiosity. Science 344: 449 (2014).

[22] Wakeford, H. R. et al. HAT-P-26b: A Neptunemass exoplanet with a well-constrained heavy element abundance. Science 356: 628-631 (2017).

[23] Weinberg, S. Dreams of a Final Theory Vintage Books: 220, 229 (1992).

[24] Weinberg, S. Facing up: Science and Its Cultural Adversaries Harvard Univ. Press: 7, 173-174, 238-239 (2003).

[25] Wilson, E. O. Letters to a Young Scientist Liveright: 125 (2013).

[26]Zhang, Q. et al. Transboundary health impacts of transported global air pollution and international trades. Nature 543: 705-709 (2017). 